\title{
Risk factors for failure of continuous positive airway pressure treatment in patients with ostructive sleep apnoea
}

\author{
David Sloukaa , Monika Honnerova ${ }^{b}$, Petr Hosek ${ }^{c}$, Ales Matas ${ }^{d}$, Karel Slama ${ }^{a}$, Jana Landsmanovae, Radek Kucera ${ }^{f}$
}

\begin{abstract}
Background and Aims. Obstructive sleep apnoea is a potentially serious sleep disorder associated with the risk of cardiovascular disease. It is treated with continuous airway pressure (CPAP) but this is not always successful. Unsuccessful cases should be treated by bilevel positive airway pressure (BiPAP). The aim of this study was to determine whether common respiratory parameters and/or body mass index (BMI) can be used to predict the probability CPAP failure and hence start such patients on BiPAP from the outset.

Methods. A sample of patients treated by CPAP for OSAS was evaluated a retrospective cohort study. The data measured in sleep monitoring of the successfully treated group and of the group where CPAP had failed were compared. Subsequently, the predictive abilities of BMI, Apnoea Index (Al), Apnoea-Hypopnea Index (AHI), percentage of sleep time in less than $90 \%$ oxygen saturation (T90), average oxygen saturation over the duration of sleep (SaO2) and average desaturation per hour of sleep (ODI) were assessed with respect to CPAP failure, both individually and in combination. Results. A sample of 479 patients was included in the study. All of the recorded variables except Al were significantly associated with failure of CPAP and their ability to predict the failure ranged from poor to moderate. Since there was significant correlation among all the variables measured a two-variable prediction model combining T90 and BMI produced no significant improvement in the quality of CPAP failure prediction.

Conclusions. BMI was a significant predictor of CPAP failure although it was slightly less predictive than T90. The set of monitored variables included in our study does not allow for CPAP failure to be predicted with clinically relevant reliability.
\end{abstract}

Key words: sleep apnoea syndrome, obesity, BMI, CPAP failure, prediction

Received: July 12, 2017; Accepted with revision: December 6, 2017; Available online: January 18, 2018 https://doi.org/10.5507/bp.2017.056

${ }^{a}$ Department of Otorhinolaryngology, Faculty of Medicine in Plzen, Charles University in Prague, Edvarda Benese 13, 30599 Pilsen, Czech Republic

${ }^{b}$ Department of Pneumology, Faculty of Medicine in Plzen, Charles University in Prague, Edvarda Benese 13, 30599 Pilsen, Czech Republic 'Biomedical Center, Faculty of Medicine in Plzen, Charles University in Prague, alej Svobody 1655/76, 32300 Pilsen, Czech Republic ${ }^{d}$ Department of Mathematics, Faculty of Applied Sciences, University of West Bohemia Pilsen, Univerzitni 2762/22, 30100 Pilsen, Czech Republic

'Department of Gynaecology, Faculty of Medicine in Plzen, Charles University in Prague, Alej Svobody 80, 30460 Pilsen, Czech Republic ${ }^{f}$ Laboratory of Immunoanalysis, Department of Nuclear Medicine, Medical School and Teaching Hospital in Pilsen, Charles University in Prague, Pilsen, Czech Republic

Corresponding author: David Slouka, e-mail: slouka@fnplzen.cz

\section{INTRODUCTION}

The obstructive sleep apnea syndrome (OSAS) is a potentially serious sleep disorder characterized by repetitive pauses in respiration or periods of reduced breathing during the sleep. In general, the population around $4 \%$ of middle-aged men and $2 \%$ of middle-aged women can be affected by OSAS (ref. ${ }^{1,2}$ ).

OSAS increases overall morbidity and mortality and it is associated with a triple increased risk of cardiovascular disease ${ }^{3,4}$. In patients with this condition, there is a greater prevalence of obesity than in the general population, according to available studies, in $40-70 \%$ of patients with OSAS (ref. ${ }^{5,6}$ ), and thus considered to be one of the respiratory complications of obesity. This fact is serious because of its increasing incidence ${ }^{7}$ with $10-25 \%$ of the European and American population having a BMI more than $30\left(\right.$ ref. $\left.^{8}\right)$.
In patients suffering from moderate and severe OSAS, positive airway pressure therapy (PAP) is the most effective treatment ${ }^{9,10}$. PAP machines deliver air to the upper airways at a pressure high enough to keep them open. The first, and most widely used choice of treatment is continuous positive airway pressure (CPAP) (ref. ${ }^{11,12}$ ). In cases of failure, this must be replaced by bilevel positive airway pressure therapy (BiPAP) $\left(\right.$ ref. $\left.{ }^{13}\right)$. Both devices operate on the same principle. The essential difference between CPAP and BiPAP is that while the former is set to a single pressure level, the BiPAP has two pressure settings: the prescribed pressure for inhalation and a lower pressure for exhalation. Patients are given proper a priori instructions on the use of the positive airway pressure device ${ }^{14,15}$. However, the CPAP treatment is still ineffective in about $13 \%$ of cases. In these patients, even though the apnoea/ hypopnoea index (AHI) drops, hyposaturation persists ${ }^{16,17}$.

Patients with CPAP failure (persistent hyposaturation 
found in routine check up - sleep monitoring in 3 months of CPAP treatment) are candidates for BiPAP therapy ${ }^{18}$. However, the latter device can only be used with evidence of the inefficacy of the former which usually takes several months. The determination of reliable factors predicting the failure of CPAP would thus enable patients to be started immediately on BiPAP therapy, obviating the inconvenience and cost associated with the temporary use of the ineffective CPAP ( ref. $^{19}$ ).

The aim of our study was to compare selected respiratory parameters and BMI for successfully and unsuccessfully CPAP treated patients to determine whether these factors predict the risk of CPAP failure.

\section{METHODS}

In this monocentric, observational, retrospective cohort study, on a sample of patients treated by CPAP for OSAS, the data measured in sleep monitoring were evaluated and compared for successful and unsuccessfully treated groups. The predictive ability of the monitored variables with respect to CPAP failure were assessed, individually and in combination.

Inclusion criteria: 1. OSAS diagnostic and control evaluation performed using the same polygraphic monitoring systems (Miniscreen 8, Vitaloc or Stardust), 2. the CPAP devices being the first choice of therapy and. 3 . good compliance with therapy.

Exclusion criteria: 1. incomplete data for measured parameters, 2. previous surgical and/or conservative treatment for OSAS, 3. chronic obstructive pulmonary disease (COPD) and, 4. alcohol abuse.

\section{Ethics}

The Ethics Committee of the University Hospital Pilsen approved the data analyses.

\section{Recorded data}

The following variables were recorded and analyzed:

- BMI (body mass index) - Body weight divided by the square of height; a measure of the degree of obesity.

- AI (apnoea index) - Total number of apnoeae during one hour.

- AHI (apnoea-hypopnea index) - Total number of apnoeae and hypopneae in the course of one hour.

- ODI (oxygen distress index) - Average desaturation per hour of sleep.

- $\mathrm{SaO} 2$ (average saturation) - Average oxygen saturation over the duration of sleep.

- T90 - Percentage of sleep time in less than 90\% oxygen saturation.

The effect of CPAP was assessed generally 3 months after its initial application.

The following values of respiratory parameters were considered as CPAP failure: i) AHI > 10 and lower than a $75 \%$ decrease of the baseline AHI value; ii) $\mathrm{AHI}<10$, but persisting hyposaturation, T90 $\geq 10 \%$ and ODI $\geq 10$ $\left(\right.$ ref. $\left.^{20}\right)$.

\section{Statistical methods}

The association between gender and CPAP failure rate was tested using Fisher's exact test. Due to the nonnormal distributions of quantitative variables, confirmed by the Shapiro-Wilk test, non-parametric test were used - differences in parameters between the successfully and unsuccessfully treated patients were tested using the Mann-Whitney U test; mutual correlations between the quantitative variables were assessed with the Spearman's correlation coefficient. All reported p-values were twotailed and the level of statistical significance was set at $\alpha$ $=0.05$. Patients with missing data were omitted from the analyses for which the data were missing and kept for the rest (pairwise deletion).

The discrimination potential of the quantitative variables with respect to CPAP failure was assessed using the Receiver Operating Characteristic (ROC). The ROC curve, describing observed sensitivity and specificity values across all possible threshold values within the range of a particular variable, was plotted for each variable. The area under the curve (AUC ROC) was then calculated to summarize the prediction factor in a single value, ranging from 1 (perfectly reliable prediction) to 0.5 (random guessing). Sensitivity and specificity were determined for specific threshold values. Confidence intervals for sensitivity, specificity and relative frequency were calculated according to Agresti and Coull ${ }^{21}$.

Despite the strong correlations found among all of the quantitative variables, a two-variable prediction model for CPAP failure was then constructed. The model employed BMI and T90 which both showed relatively solid ROC results and moderate correlation. The model was implemented by a simple threshold-based classification upon a new variable obtained as a linear combination of T90 and BMI the coefficients of which were optimised to maximise the area under the ROC curve. The optimisation was carried out by an enumerative exploration of the whole possible range of rotation angles of the combined factor with respect to the coordinate system defined by the original variables.

The STATISTICA data analysis software system (StatSoft, Inc.2013. Version 12. www.statsoft.com) was used; the ROC analysis and optimisation of the two-variable model were carried out in MATLAB (Release 2014b, The MathWorks, Inc., Natick, MA, USA)

\section{RESULTS}

From 2005 to 2010, a total of 983 patients with SAS were examined at the Department of Otolaryngology, University Hospital in Pilsen. 479 (338 male and 141 female) of these met the inclusion criteria and were included in the study. The mean age was $58.3 \pm 11.3$ (SD) years.

The rate of CPAP failure observed in the whole sample was $12.5 \%$ (95\% confidence interval 9.8-15.9\%). There was no significant gender difference.

With the exception of AI $(P=0.1785)$, all of the quantitative variables were significantly associated with 
A

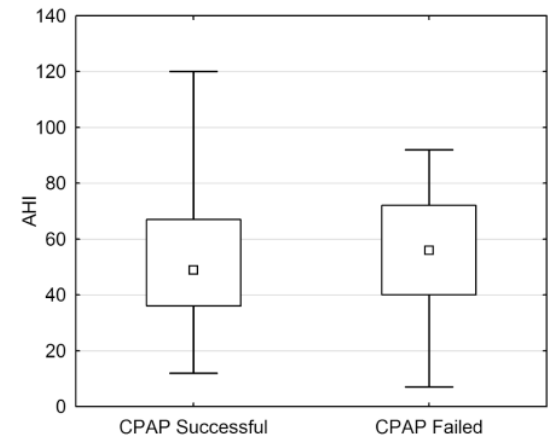

D

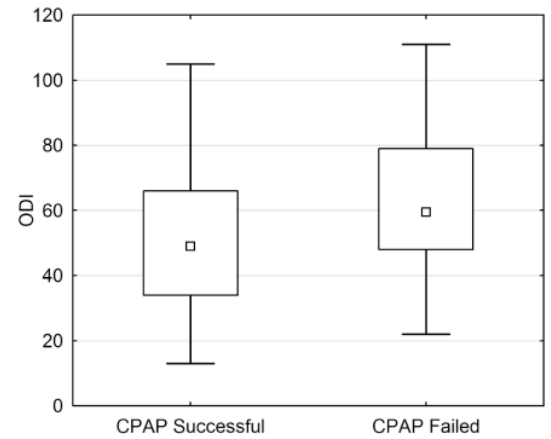

B

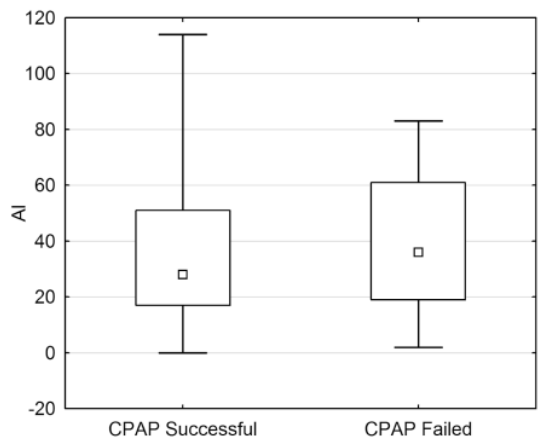

$E$

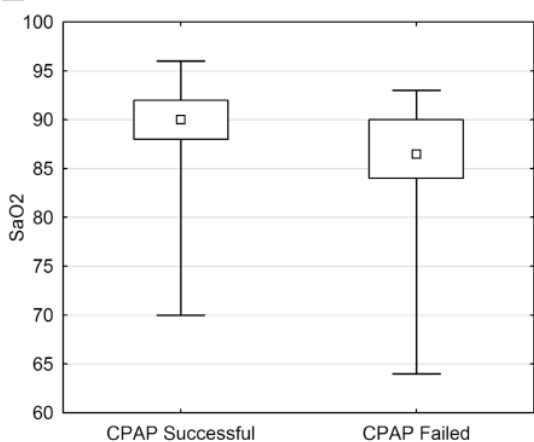

C

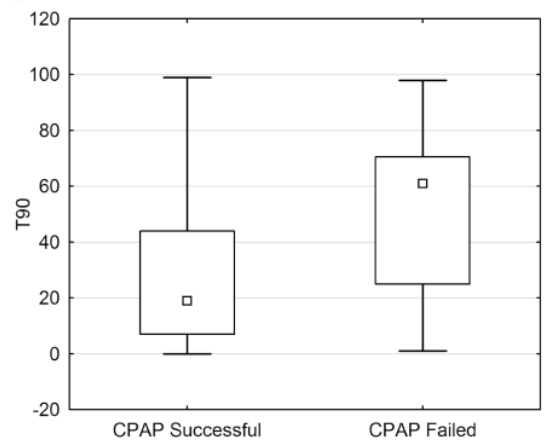

$\mathrm{F}$

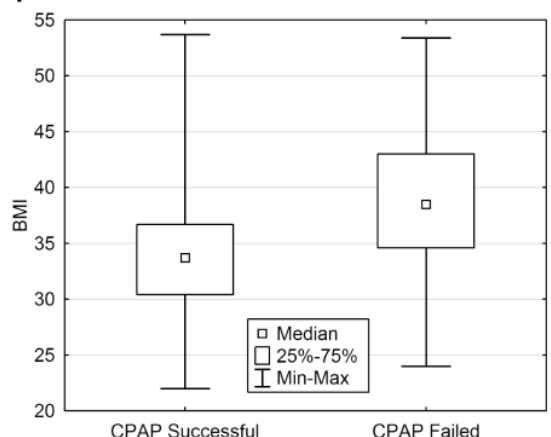

Fig. 1. Comparison of the monitored variables between the patients with successful and unsuccessful CPAP therapy. A - AHI, B - AI, C - T90, D - ODI, E - SaO2, F - BMI.

the success/failure of CPAP (Fig. 1, AHI: $P=0.0172$, T90: $P<0.0001$, ODI: $P=0.0002, \mathrm{SaO} 2: P<0.0001$, BMI: $P<0.0001)$. Significant mutual correlations of all variables, in particular between $\mathrm{T} 90$ and $\mathrm{SaO} 2$ and between $\mathrm{AHI}$ and AI, were found (all $P<0.05$, see Table 1).

Table 1. Mutual correlations between the monitored variables. Spearman's correlation coefficients are shown; all reported correlations were statistically significant.

\begin{tabular}{lcccccc}
\hline & AHI & \multicolumn{1}{c}{ AI } & \multicolumn{1}{l}{ T90 } & ODI & SaO2 & BMI \\
\hline AHI & & 0.828 & 0.519 & 0.778 & -0.451 & 0.283 \\
AI & 0.828 & & 0.468 & 0.661 & -0.403 & 0.147 \\
T90 & 0.519 & 0.468 & & 0.651 & -0.913 & 0.454 \\
ODI & 0.778 & 0.661 & 0.651 & & -0.590 & 0.397 \\
$\mathrm{SaO} 2$ & -0.451 & -0.403 & -0.913 & -0.590 & & -0.470 \\
BMI & 0.283 & 0.147 & 0.454 & 0.397 & -0.470 & \\
\hline
\end{tabular}

According to the ROC analysis (Fig. 2), T90 showed the best albeit moderate discrimination ability for CPAP failure prediction (AUC ROC curve 0.755). T90 was closely followed by $\mathrm{SaO} 2$ (AUC ROC 0.732), BMI (AUC ROC 0.695) and ODI (AUC ROC 0.646). AHI and AI with AUC ROC values below 0.6 appeared to be not good predictive factors. The strongest factor - T90 - exhibited, at an empirically determined threshold value of 43.5 , a sensitivity of $68.3 \%$ (95\% confidence interval $55.5-78.9 \%$ ) and a specificity of $74.5 \%$ (70.0-78.5\%).

The two-variable model for CPAP failure prediction was constructed using T90 (the best performing factor in ROC) and BMI (the third best performance in ROC). $\mathrm{SaO} 2$, the second best factor according to ROC, was omitted because of its strong correlation with T90, and the employment of BMI was also of the highest clinical interest. After optimization, the model exhibited a value of AUC ROC of 0.761 , thus showing only negligible improvement over the prediction based on T90 alone (AUC ROC 0.755).

\section{DISCUSSION}

The results showed that obesity as measured by BMI was a significant risk factor for CPAP therapy failure. The comparison and assessment of other measured variables showed that the majority of respiratory parameters were significantly worse in patients where CPAP had failed. To our present knowledge, this study is so far the largest one assessing the interplay between obesity and respiratory parameters with respect to CPAP failure.

Even though there is a dearth of information in the literature, a similar study involving a smaller group of 146 patients was published by Schafer et al. ${ }^{22}$ (1998), reporting an association between T90, BMI and CPAP failure. In accordance with our results, these authors found that obesity was a strong risk factor for CPAP failure, with median BMI pf 31 in successfully, and 44 in unsuccessfully treated patients.

Even though our results showed that BMI, as well as AHI, ODI, T90 and $\mathrm{SaO} 2$ were significant risk factors for CPAP failure, the ROC analysis suggests that none of these variables alone or in combination (as demonstrated 

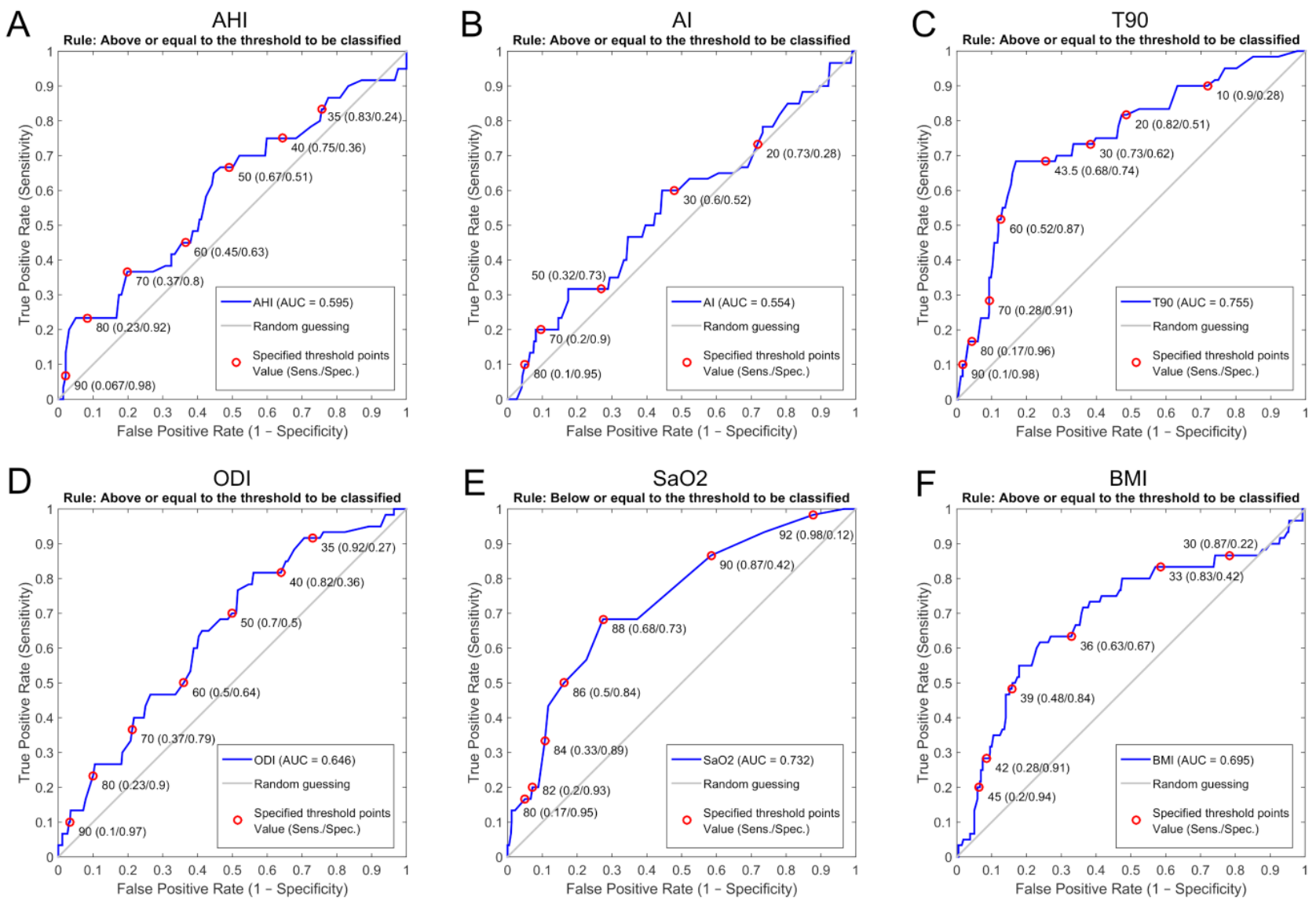

Fig. 2. ROC analysis of the monitored variables with respect to the prediction of CPAP failure. A - AHI, B - AI, C - T90, D ODI, E - SaO2, F - BMI. Cases compliant with the rule stated above each plot were predicted to fail in CPAP.

by the two-variable model) can be used for the predicting CPAP failure with clinically relevant reliability. If T90, which was the best prediction parameter, were used at the suggested empirical threshold value of 44 to make the decision about the indication of BiPAP instead of CPAP, the observed sensitivity and specificity values $(68 \%$ and $75 \%$, respectively) together with the prevalence of CPAP failure (13\%) would result in $72 \%$ of the BiPAP treatments to be indicated unnecessarily (i.e. positive predictive value of $28 \%$ ), while, almost a third of actual CPAP failures would not be predicted. However, considering the high negative predictive value of T90 (94\%), the relevance of this prediction criterion is considerably better, as T90 below 44 contradicts the possibility of CPAP failure with almost $95 \%$ reliability.

Significant correlations between BMI and all of the respiratory parameters not only explain the failure of the two-variable model to improve the prediction, they also suggest the underlying mechanism of CPAP failure which is generally more likely to occur in more severe cases of OSAS. Rather than affecting the CPAP treatment results directly, the high BMI can aggravate the general body condition of the patient, which consequently can lead to the worse response to the CPAP therapy.

Significant mutual correlations among the respiratory parameters measured indicate mutual relations between these variables. In particular, the strong association between $\mathrm{AI}$ and AHI as well as between $\mathrm{SaO} 2$ and T90 show their mutual redundancy as both variables in each pair carry virtually identical information. AI is no longer used because of its poor clinical relevance; $\mathrm{SaO} 2$ is still routinely monitored in some clinics.

Even though an accurate indication of BiPAP as the first-choice device in the cases where CPAP would fail would provide substantial advantage to patients as well as a considerable cost reduction of the treatment, current commonly monitored variables, unfortunately, do not allow for such prediction to be made reliably.

Our study is limited by its retrospective and monocentric design. Nonetheless, this limitation is not supposed to be very serious as the large number of patients enrolled and the demographic distribution support its validity. Moreover, demographic data in our work correspond to those of one large study comprising more than twelve thousand individuals ${ }^{23}$.

\section{CONCLUSIONS}

AHI, AI, T90, ODI, SaO2 and BMI are commonly recorded parameters in the diagnostics of OSAS. With the exception of AI, they were proven as significant risk factors of the failure of CPAP therapy. T90, $\mathrm{SaO} 2$ and BMI showed a moderate ability to predict CPAP failure, although with clinically insufficient reliability. However, even though T90 cannot satisfyingly predict CPAP failure, 
it can eliminate it with reasonable accuracy. Our results also show that all of the factors are strongly mutually dependent, which makes their simultaneous inclusion in the decision redundant.

\section{ABBREAVIATIONS}

AHI, Apnoea-hypopnoea index; AI, Apnoea index; AUC, Area under the curve; BiPAP, Bilevel positive airway pressure; BMI, Body mass index; CPAP, Continuous positive airway pressure; PAP, Positive airway pressure; ODI, Oxygen distress index; OSAS, Obstructive sleep apnoea syndrome; ROC, Receiver operating characteristic; $\mathrm{SaO} 2$, Average saturation; T90, Percentage of sleep time in less than $90 \%$ oxygen saturation.

Acknowledgement: This project was supported by the grant of Ministry of Health of the Czech Republic Conceptual Development of Research Organization (Faculty Hospital in Pilsen - FNP1, 00669806), and by the National Sustainability Program I (NPU I) Nr. LO1503 provided by the Ministry of Education Youth and Sports of the Czech Republic.

Author contributions: DS: conception, manuscript writing, literature search, final approval; $\mathrm{MH}$ : literature search, final approval; PH: conception, statistical analysis; AM: statistical analysis; KS: literature search; RK: literature search.

Conflict of interest statement: The authors state that there are no conflicts of interest regarding the publication of article.

Ethics: The Ethics Committee of the University Hospital Pilsen granted their approval to the data analyses.

\section{REFERENCES}

1. Hobzova M, Salzman R, Stejskal D. Serum adiponectin level in obstructive sleep apnea: Relation of adiponectin to obesity and longterm continuous positive airway pressure therapy. Adv Med Sci 2016;61(1):130-4.

2. Mannarino MR, Di Filippo F, Pirro M. Obstructive sleep apnea syndrome. Eur J Intern Med 2012;23(7):586-93.

3. Monahan K, Redline S. Role of obstructive sleep apnea in cardiovascular disease. Curr Opin Cardiol 2011;26(6):541-7.
4. Kasai T. Sleep apnea and heart failure. J Cardiol 2012;60(2):78-85.

5. Aguiar IC, Freitas WR Jr, Santos IR. Obstructive sleep apnea and pulmonary function in patients with severe obesity before and after bariatric surgery: a randomized clinical trial. Multidiscip Respir Med 2014;9(1):43.

6. Ernst G, Bosio M, Salvado A. Difference between apnea-hypopnea index (AHI) and oxygen desaturation index (ODI): proportional increase associated with degree of obesity. Sleep Breath 2015;4:245-9.

7. Block JP, Oken E. Practical Considerations for the US Preventive Services Task Force Recommendations on Obesity in Children and Adolescents. JAMA Intern Med 2017 Jun 20;64(5):392-406.

8. Braunerova R, Hainer V. Obesity. Diagnostics and treatment. Med Pro Praxi 2010;7(1):19-22.

9. Epstein LJ, Kristo D, Strollo PJ Jr et al. Clinical guideline for the evaluation, management and longterm care of obstructive sleep apnea in adults. J Clin Sleep Med 2009;5(3):263-76.

10. Lojander J, Maasilta P, Partinen M. Nasal-CPAP, surgery, and conservative management for treatment of obstructive sleep apnea syndrome. Chest 1996;110:114-9.

11. Butner KL, Hargens TA, Kaleth AS. Association of Obstructive Sleep Apnea Severity with Exercise Capacity and Health-related Quality of Life. N Am J Med Sci. 2013;5(6):362-6.

12. Chowdhury O, Wedderburn CJ, Duffy D. CPAP review. Eur J Pediatr 2012;171(10):1441-8.

13. Piper AJ, Wang D, Yee BJ. Randomised trial of CPAP vs bilevel support in the treatment of obesity hypoventilation syndrome without severe nocturnal desaturation. Thorax 2008;63(5):395-401.

14. La Piana GE, Scartabellati A, Chiesa L. Long-term adherence to CPAP treatment in patients with obstructive sleep apnea: importance of educational program. Patient Prefer Adherence 2011;5:555-62.

15. Williams B, Boyle M, Robertson N. When Pressure is Positive: A Literature Review of the Prehospital Use of Continuous Positive Airway Pressure. Prehosp Disaster Med 2013;28(1):52-60.

16. Krieger J, Weitzenblum E, Monassier J. Dangerous hypoxaemia during continuous positive airway pressure treatment of obstructive sleep apnoea. Lancet 1983;2:1429-30.

17. Mulgrew AT, Lawati NA, Ayas NT. Residual sleep apnea on polysomnography after 3 months of CPAP therapy: clinical implications, predictors and patterns. Sleep Med 2010; 11(2):119-25.

18. Dohi T, Kasai T, Narui K. Bi-level positive airway pressure ventilation for treating heart failure with central sleep apnea that is unresponsive to continuous positive airway pressure. Circ J 2008 Jul;72(7):1100-5.

19. Billings ME, Kapur VK. Medicare long-term CPAP coverage policy: a cost-utility analysis. J Clin Sleep Med 2013;15;9(10):1023-9.

20. M. Pretl, M. Hobzova, M. Honnerova. The criteria for the CPAP treatment in sleep disorders. Guidelines 2011, Czech Sleep Research and Sleep Medicine Society, http://www.sleep-society.cz/index.php/en/

21. Agresti A, Coull BA. Approximate is Better than "exact" for interval estimation of binomial proportions. The American Statistician 1998;52:119-26.

22. Schäfer H, Ewig S, Hasper E. Failure of CPAP therapy in obstructive sleep apnoea syndrome: predictive factors and treatment with bilevel-positive airway pressure. Respir Med. 1998;92(2):208-15.

23. Vozoris NT. Sleep apnea-plus: prevalence, risk factors, and association with cardiovascular diseases using United States populationlevel data. Sleep Med 2012;13(6):637-44. 